\title{
Modelica Component Models for Non-diffracting Floating Objects and Quasi-static Catenary Moorings
}

\author{
Savin Viswanathan ${ }^{1}$ Christian Holden ${ }^{1}$ \\ ${ }^{1}$ Dept. of Mechanical and Industrial Engineering, Norwegian University of Science and Technology (NTNU), \\ NO-7491 Trondheim, Norway. \{savin.viswanathan, christian.holden\}@ntnu. no
}

\begin{abstract}
In this paper, the theory behind determining the hydrodynamic response of a floating object in the presence of waves is discussed, followed by a simplification for the case of wave-transparent objects. The Morison equation is introduced as a means to estimate lateral wave and current loads on slender bodies. The quasi-static catenary approach to determine mooring forces is then discussed. Development of Modelica component-models to simulate the hydrodynamic response of free-floating and catenarymoored non-diffracting objects, in the presence of waves and depth varying current, is then dealt with in detail, and the results dicussed.

Keywords: hydrodynamics of non-diffracting floating objects, quasi-static catenary mooring, Modelica oceanengineering library.
\end{abstract}

\section{Introduction}

The advantages of developing an OpenModelica oceanengineering library populated with domain-specific component-models and functions to carry out the integrated simulation of multi-pyhsical ocean engineering systems was demonstrated by the authors (Viswanathan and Holden, 2019). This earlier work:

1. Gives a brief description of the simulation of systems based on the hydrodynamic response of catenarymoored non-diffracting floating objects in the presence of waves and current,

2. Demonstrates the satisfactory agreement of the Modelica simulation results with those obtained using a popular ocean-engineering commercial software (Orcaflex), and

3. Brings out the advantages of using a componentmodel based simulation approach.

The voluminous nature of the earlier work precluded the possibility of delving into the theoretical and implementational details of the various Modelica componentmodels of the ocean-engineering library proposed by the authors, the preliminary version of which is available for download at github.com/Savin-Viswanathan/ OELib_OMAE2019.
(Viswanathan and Holden, 2020) gives a detailed description of the development of Modelica componentmodels to simulate regular as well as irregular waves and depth-varying current. This present work elaborates on the theoretical and implementational details of the component-models for non-diffracting floating objects, and catenary mooring based on the quasi-static approach.

\section{Theory}

\subsection{Hydrodynamics}

Considering the steady-state interaction of a floating object with a regular wave, the loads acting on the body may be considered to be comprised of:

- The fluid pressure loads due to the incident wave acting on the body, which is assumed fixed at its mean position.

- The fluid pressure loads due to the scattered/diffracted wave from the body, which is assumed fixed at its mean position.

- The fluid pressure loads due to the radiated wave system set up by the body as it oscillates in its six Degrees-of-Freedom (DoF) in calm water.

An illustration of the Diffraction-Radiation problem is given in Figure 1.

The loads due to the incident wave are referred to as Froude-Krylov loads, and those due to the scattered wave are referred to as diffraction loads in p. 39 of (Faltinsen, 1999), and this is the convention followed in this work. Another widely used convention is to refer to the combined incident and scattered wave-problem as the diffraction problem as in p. 288 of (Newman, 1989).

The Froude-Krylov and diffraction loads taken together constitute the wave excitation loads and may be determined by integrating the incident and diffracted wave dynamic pressures over the mean wetted hull surface. The integration of the dynamic component of the radiation wave pressures give the associated hydrodynamic loads commonly referred to as added-mass and damping, while the integration of the hydrostatic component gives the restoring loads. The added-mass loads are in phase with the body acceleration and the damping loads are in phase with the body velocity. 

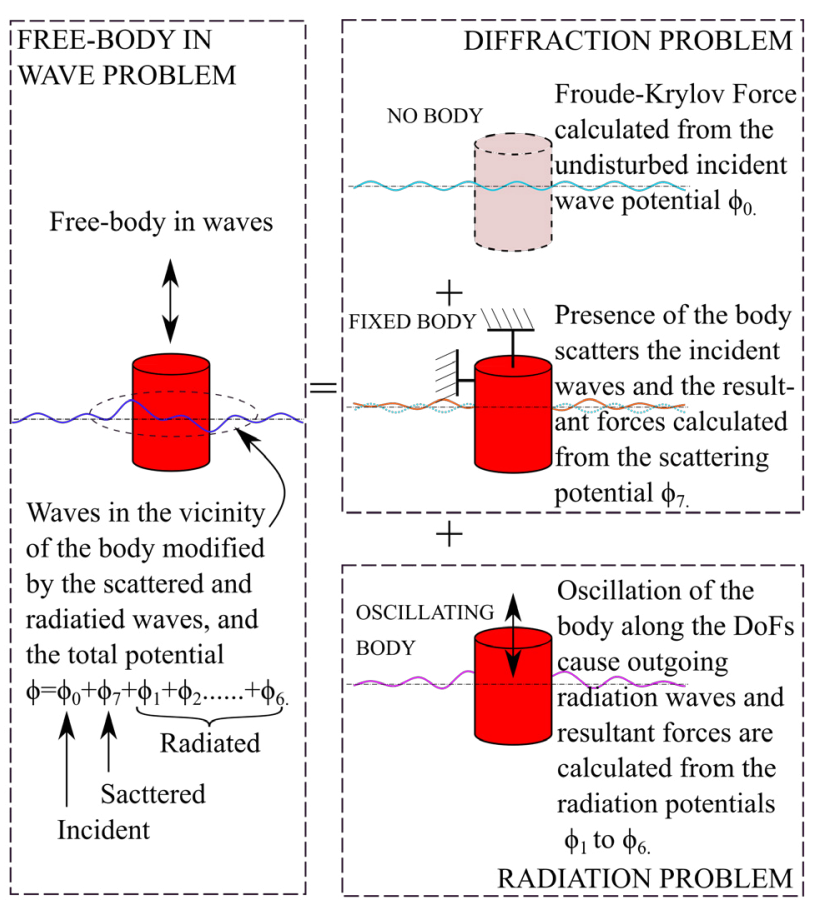

Figure 1. The diffraction-radiation problem. Adapted from (Faltinsen, 1999)

The assumption of inviscid, incompressible fluid and irrotational flow implies the existance of a velocity potential which may be determined by solving the associated linearized boundary value problem (BVP). The solution to the BVP for determining the incident wave potential is discussed in (Viswanathan and Holden, 2020). Each of the wave systems above may thus be described by its respective velocity potential, and within the assumption of linearity, the total potential $\phi\left[\mathrm{m}^{2} / \mathrm{s}\right]$ in the vicinity of the body is given by

$$
\phi=\phi_{0}+\phi_{7}+\sum_{j=1}^{6} \dot{\eta}_{j} \varphi_{j}
$$

Here, $\phi_{0}$ and $\phi_{7}\left[\mathrm{~m}^{2} / \mathrm{s}\right]$ are the incident and diffracted wave velocity potentials, respectively, and the summation term represents the radiation wave potential, where $\dot{\eta}_{j}[\mathrm{~m} / \mathrm{s}]$ is the body velocity along the respective DoF, and $\varphi_{j}[\mathrm{~m}]$ is the spatial component of the complex velocity-potential due to the body oscillation with unit velocity in the corresponding DoF; see (Faltinsen and Michelsen, 1974).

Numerical solutions to the diffraction-radiation problem may be sought through the Boundary Element Method or through the Harmonic Polynomial Cell method, to determine the hydrodynamic coefficients. See (Newman and Lee, 2002) and (Shao and Flatinsen, 2014).

\subsubsection{Simplifications in the case of a small-volume structure}

When the size of the structure is large, the diffraction forces are significant, and hence, one must solve the diffraction-radiation problem to determine the wave loads. However, when the structure is relatively small compared to the incident wave-length, depending on the relative significance of the inertia and drag forces, one may utilize the Froude-Krylov theory or the Morison equation to determine the wave loads. The Froude-Krylov theory is applicable for a relatively small structure when drag forces are small compared to the inertia forces. When the drag forces are significant, one has to use the Morison equation; see p. 168 of (Chakrabarti, 1987).

Considering the case of a vertical cylindrical buoy, drag forces are not signifcant for motions in the vertical plane, and hence the Froude-Krylov theory can be used to calculate the vertical wave-loads. However, for motion in the horziontal plane, drag forces become significant and hence, the Morison equation should be used to determine the horizontal wave-loads.

2.1.1.1 The Froude-Krylov Force For small-volume upright cylindrical structures, the long wave approximation is applicable for $L>5 D$, where $L[\mathrm{~m}]$ is the wavelength and $D[\mathrm{~m}]$ is the diameter of the cylinder. Considering the translational DoFs, i.e., $i=1,2,3$, the force on the relatively small body may then be expressed as:

$$
\begin{aligned}
\mathbf{F} & =\mathbf{i} F_{1}+\mathbf{j} F_{2}+\mathbf{k} F_{3} \\
\text { where } F_{i} & =-\iint_{S_{0 B}} p n_{i} \mathrm{~d} s+A_{i 1} a_{1}+A_{i 2} a_{2}+A_{i 3} a_{3} .
\end{aligned}
$$

Here, $p\left[\mathrm{~N} / \mathrm{m}^{2}\right]$ is the undisturbed incident wave pressure, and $\mathbf{n}=\left(n_{1}, n_{2}, n_{3}\right)$ is the unit normal vector to the body surface, defined to be positive into the fluid. The integral is over the average wetted surface of the body. Furthermore, $a_{1}, a_{2}$, and $a_{3}\left[\mathrm{~m} / \mathrm{s}^{2}\right]$ are the acceleration components along the $x, y, z$ directions of the undisturbed wave field, and are to be evaluated at the geometrical mass centre of the body. $A_{i 1}, A_{i 2}, A_{i 3}[\mathrm{~kg}]$ are added-mass terms. $\mathbf{i}, \mathbf{j}, \mathbf{k}$ are unit vectors along $x, y, z$. We also note that the wave generation capability of the body is very small when the long-wave approximation holds true, and hence, the potential damping terms may be neglected; see pp. 60-61 of (Faltinsen, 1999).

The first term of (3) is the Froude-Krylov force, the vertical component of which is approximated as

$$
F_{F K}^{z} \approx \rho g A_{w p} \eta .
$$

Here, $\rho\left[\mathrm{kg} / \mathrm{m}^{3}\right]$ is the water density, $g\left[\mathrm{~m} / \mathrm{s}^{2}\right]$ is the acceleration due to gravity, $A_{w p}\left[\mathrm{~m}^{2}\right]$ is the water-plane area, and $\eta[\mathrm{m}]$ is the sea surface elevation (SSE) about the mean sea level. See (Techet, 2005).

The sea surface elevation $\eta$, at any $x$ co-ordinate, may be expressed as:

$$
\eta(x, t)=\sum_{i=1}^{N} \zeta_{0 i} \cos \left(k_{i} x-\omega_{i} t-\varepsilon_{i}\right) .
$$

Here, $N$ is the total number of wave components (frequency bands), $\zeta_{0 i}[\mathrm{~m}]$ is the component wave amplitude, 
$\omega_{i}[\mathrm{rad} / \mathrm{s}]$ is the wave angular frequency, and $\varepsilon_{i}[\mathrm{rad}]$ is the phase. Subscript $i$ refers to the number of the componentwave under consideration. The wave number $k_{i}[\mathrm{rad} / \mathrm{m}]$ is to be determined from the dispersion relation. For details, see (Viswanathan and Holden, 2020).

The SSE for a regular wave can be expressed by taking $N=1$ in (5).

2.1.1.2 The Morison Equation Though initially formulated to calculate the horizontal wave-forces on fixed, surface-piercing vertical piles, where $D \ll L$, and the drag forces significant, the Morison equation has since been adapted to determine wave loads on oscillating slender structures. A thorough treatment of the subject can be found in Chapter 6 of (Chakrabarti, 1987).

The horizontal wave and current loads per unit length, on a cylindrical object free to move in presence of waves and current, may be determined from

$$
\begin{aligned}
M_{F}^{x}= & C_{M}^{x} \rho \frac{\pi}{4} D^{2} \dot{u}-C_{A}^{x} \rho \frac{\pi}{4} D^{2} \ddot{x} \\
& +C_{D}^{x} \frac{1}{2} \rho D|u \pm U-\dot{x}|(u \pm U-\dot{x}) .
\end{aligned}
$$

Here, $M_{F}^{x}[\mathrm{~N}]$ is the Morison force, $C_{M}^{x}[-]$ is the inertia coefficient, $\rho\left[\mathrm{kg} / \mathrm{m}^{3}\right]$ is water density, $D[\mathrm{~m}]$ is the body diameter, $\dot{u}\left[\mathrm{~m} / \mathrm{s}^{2}\right]$ is the wave-induced water-particle acceleration along $x, C_{A}^{x}[-]$ is the added-mass coefficient, $\ddot{x}$ $\left[\mathrm{m} / \mathrm{s}^{2}\right]$ is the body acceleration along $x, C_{D}^{x}[-]$ is the drag coefficient, $u[\mathrm{~m} / \mathrm{s}]$ is the wave-induced water-particle velocity along $x, U[\mathrm{~m} / \mathrm{s}]$ is the current velocity along $x$, and $\dot{x}[\mathrm{~m} / \mathrm{s}]$ is the body velocity along $x . C_{M}^{x}$ and $C_{D}^{x}$ are available from numerous field and laboratory tests, e.g., (Yeung, 1981), which allows the designer to choose appropriate values; see p. 172 of (Chakrabarti, 1987). Also, $C_{M}^{x}=1+C_{A}^{x}$; see p. 178 of (Chakrabarti, 1987). The wave kinematics are given by (7) and (8); see pp. 48-52 of (Chakrabarti, 1987).

$$
\begin{aligned}
& u=\frac{\pi H}{T} \frac{\cosh [k(z+d)]}{\sinh k d} \cos (k x-\omega t) \\
& \dot{u}=\frac{2 \pi^{2} H}{T^{2}} \frac{\cosh [k(z+d)]}{\sinh k d} \sin (k x-\omega t) .
\end{aligned}
$$

Here, $H[\mathrm{~m}]$ is the wave height, $T[\mathrm{~s}]$ is the wave period, $k$ $[\mathrm{rad} / \mathrm{m}]$ is the wave number, $z[\mathrm{~m}]$ is the vertical coordinate of the point at which the wave kinematics are to be calculated, $d[\mathrm{~m}]$ is the water depth, $x[\mathrm{~m}]$ is the horizontal coordinate of the point at which the wave kinematics are to be calculated, and $\omega[\mathrm{rad} / \mathrm{s}]$ is the angular frequency of the wave.

\subsection{Catenary Mooring}

When a floating object is moored by a slack mooring line, the line assumes the shape of a half catenary; see p. 9 of (Chakrabarti, 1987).

For simplicity, we consider a mooring line that acts in the $x$ - $z$ plane. At the point of suspension, the chain tension has a horizontal and vertical component, the magnitudes of which depend on $\psi$ [rad], the angle made by the tangent to the caternary with the horizontal, at the point of suspension. The horizontal component of this force prevents the drifting of the floater in the direction away from the anchor position. In the absence of other external forces, the floater drifts to a position such that the suspended length of the mooring line is vertical. $X[\mathrm{~m}]$ is the distance from the anchor point to the fairlead on the buoy, and $x[\mathrm{~m}]$ is the distance from the touch- down-point (TDP) to the fairlead. As the buoy drifts away from the anchor, the TDP moves towards the anchor, and vice-versa, as shown in Figure 2. The total chain length is represented by $l_{c}[\mathrm{~m}]$ and the suspended length of the chain is represented by $l_{s}$ [m]. The length of the chain lying on the seafloor is thus $l_{f}=l_{c}-l_{s}$.

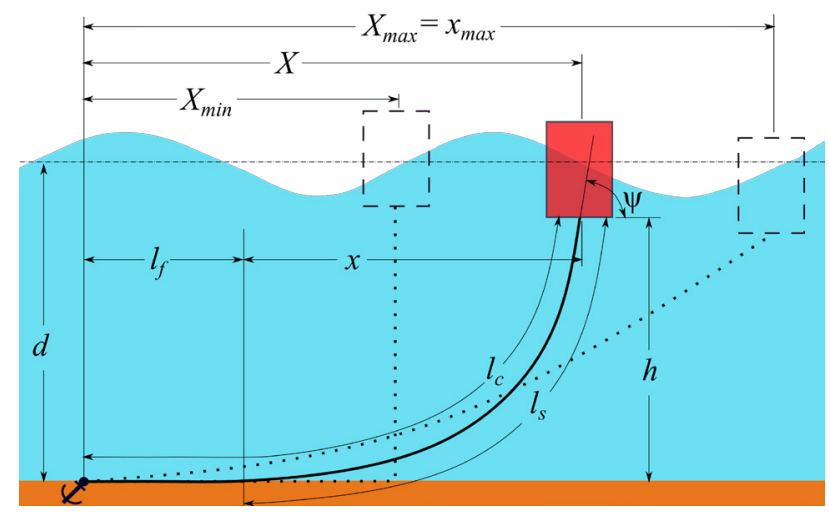

Figure 2. The mooring half-catenary.

From (Tatum, 2004) we have the following relations:

$$
\begin{aligned}
a & =\frac{T_{H}}{w} \\
z & =a \cosh \left(\frac{x}{a}\right) \\
l_{s} & =a \sinh \left(\frac{x}{a}\right) \\
z & =a \sec (\psi)=a+h \\
z^{2} & =l_{s}^{2}+a^{2} .
\end{aligned}
$$

Here, $a[\mathrm{~m}]$ is the catenary parameter, $x, z[\mathrm{~m}]$ are catenary co-ordinates, $T_{H}[\mathrm{~N}]$ is the horizontal tension, and $w[\mathrm{~N} / \mathrm{m}]$ is the submerged specific weight of the catenary.

From (MIT, 2011), we have:

$$
\begin{aligned}
T_{H} & =\frac{x w}{\cosh ^{-1}\left(1+\frac{w h}{T_{H}}\right)} \\
l_{s} & =h \sqrt{\left(1+\frac{2 T_{H}}{w h}\right)} .
\end{aligned}
$$

Considering that we are dealing with the response to linear waves of small-volume structures with small draughts and even smaller variations in draughts, and that the water depth is very large when compared to the 
draught, we note that when $X=X_{\min } \approx\left(l_{c}-d\right), x \approx 0$, and when $X=X_{\text {max }} \approx \sqrt{l_{c}^{2}-d^{2}}, x=x_{\text {max }}$.

Equation (14) can be iterated to get values of $T_{H}$ for $x \in\left\{0,0.1,0.2 \ldots \ldots x_{\max }\right\}$. We may then use the relation

$$
X=l_{c}-l_{s}+x
$$

to generate a look-up table of horizontal tension values for different horizontal positions of the floater w.r.t. the anchor position. The horizontal tensions for intermediate positions may then be determined by interpolation.

Once the instantaneous horizontal tension values are determined, we may use (10) and (12) to calculate the instantaneous suspended length of the chain, the submerged weight of which is the vertical tension at the fair lead.

The mooring line is also subject to Morison forces, both in the horizontal and vertical directions. To determine these forces, the chain is discretized into a number of segments, and the horizontal and vertical Morison loads are calculated at the mid-points of such segments, and summed up to get the loading on the entire chain. It is assumed that the catenary shape is not affected by such loads, and the sole effect of the fluid loading is a modification in the horizontal and vertical tension values for a given configuration.

The Morison loads per unit length in the normal and tangential directions to a segment, at its mid point, is calculated using equations (17) and (18). This method allows for the separate specification of $C_{D}$ and $C_{M}$ values, experimental values of which are scarce when the structures are inclined; see p. 205 of (Chakrabarti, 1987). This method is widely used, and is referred to as the cross-flow principle in p. 166 of (Orcina, 2010).

$$
\begin{aligned}
M_{F}^{n}= & C_{M}^{n} \rho \frac{\pi}{4} D^{2} a_{w}^{n}-C_{A}^{n} \rho \frac{\pi}{4} D^{2} a_{l}^{n} \\
& +C_{D}^{n} \frac{1}{2} \rho D\left|v_{w}^{n} \pm U^{n}-v_{l}^{n}\right|\left(v_{w}^{n} \pm U^{n}-v_{l}^{n}\right) . \\
M_{F}^{t}= & C_{M}^{t} \rho \frac{\pi}{4} D^{2} a_{w}^{t}-C_{A}^{t} \rho \frac{\pi}{4} D^{2} a_{l}^{t} \\
& +C_{D}^{t} \frac{1}{2} \rho D\left|v_{w}^{t} \pm U^{t}-v_{l}^{t}\right|\left(v_{w}^{t} \pm U^{t}-v_{l}^{t}\right) .
\end{aligned}
$$

Here, superscripts $n$ and $t$ denote the normal and tangential directions, and subscripts $w$ and $l$ denote the waterparticle and the mooring-segment. Further, $a\left[\mathrm{~m} / \mathrm{s}^{2}\right]$ is the acceleration, $v$ is the velocity, $U[\mathrm{~m} / \mathrm{s}]$ is the magnitude of the current velocity, and the equivalent-line diameter is $D=1.8 d_{c w}[\mathrm{~m}]$, where $d_{c w}[\mathrm{~m}]$ is the diameter of the chain wire. See p. 303 of (Orcina, 2010).

The determination of the position of the link mid-points would require the approximation of the quasi-static catenary shape from the horizontal tension at each time step. This is effected by discretizing the mooring length $l_{c}$ into a number of segments, and determining the position of each node connecting the segments using (10)-(12). Once the mid-point positions at each time step are located, the link velocity and accelerations can be expressed as time derivatives of the displacement.
The wave-induced water-particle kinematics at the link mid-point maybe calculated from (7), (8), (19), and (20); see pp. 48-52 of (Chakrabarti, 1987):

$$
\begin{aligned}
& w=\frac{\pi H}{T} \frac{\sinh [k(z+d)]}{\sinh (k d)} \sin (k x-\omega t) \\
& \dot{w}=-\frac{2 \pi^{2} H}{T^{2}} \frac{\sinh [k(z+d)]}{\sinh (k d)} \cos (k x-\omega t) .
\end{aligned}
$$

The current velocities at the required positions can be interpolated from the specified current profile. The waveinduced kinematics and the current profile are moved with the SSE. See p. 221 of (SINTEF, 2014).

Once $M_{F}^{n}$ and $M_{F}^{t}$ are determined for each link, they are resolved into their horizontal and vertical components, and summed up, to get the total horizontal and vertical forces acting on the mooring chain at each time step. These are then summed up with the vertical and horizontal mooring tension values to get the modified values with fluid loading as $F_{M}^{x}$ and $F_{M}^{z}[\mathrm{~N}]$.

\subsection{The Equations of Motion}

Having determined the loads acting on the cylindrical buoy, we may express the equations of motion (EoM) in the horizontal and vertical directions as:

$$
\begin{aligned}
M^{x} \ddot{x}+C^{x} \dot{x}+K^{x} x & =M_{F}^{x}+F_{M}^{x} \\
M^{z} \ddot{z}+C^{z} \dot{z}+K^{z} z & =F_{F K}^{z}+m_{a}^{z} \dot{w}_{c b}+F_{M}^{z}
\end{aligned}
$$

Here $M[\mathrm{~kg}]$ is mass, $C[\mathrm{Ns} / \mathrm{m}]$ is the damping, $K[\mathrm{~N} / \mathrm{m}]$ is the restoring force, $M_{F}$ is the Morison load on the buoy, $F_{M}[\mathrm{~N}]$ is the mooring load, $m_{a}[\mathrm{~kg}]$ is the added-mass, and $\dot{w}_{c b}\left[\mathrm{~m} / \mathrm{s}^{2}\right]$ is the vertical wave induced water particle acceleration evaluated at the vertical centre of buoyancy of the buoy. Superscripts $x$ and $z$ denote the horizontal and vertical directions. In (21), the added mass load is included in the Morison force term given by (6).

\section{Modelica Implementation}

Flow-charts in the sub-sections that follow have been prepared with ocean engineers, most likely to be unfamiliar with Modelica, in mind, and some elements might appear superfluous to the Modelica savvy reader.

The general considerations in the implementation of Modelica component-models for integrated simulation of ocean engineering systems, the implementation of component-models to simulate waves and depth-varying current, and the method of linking the generated outputs to a universal data bus have been discussed in (Viswanathan and Holden, 2020).

The following data are thus available at the EnvironmentBus:

- $\omega\left[{ }_{n} \omega_{i}\right]$, vector of component wave frequencies.

- $T\left[{ }_{n} \omega_{i}\right]$, vector of component wave Time periods.

- $k\left[{ }_{n} \omega_{i}\right]$, vector of component wave numbers. 
- $\varepsilon\left[{ }_{n} \omega_{i}\right]$, vector of component wave phases.

- $\zeta_{0 i}\left[{ }_{n} \omega_{i}\right]$, vector of component wave amplitudes.

- $z c g[n]$, vector of $z$ co-ordinates where current velocities are provided.

- $U \operatorname{cg}[n]$, vector of current velocities at above $z$ coordinates.

\subsection{Non-diffracting Floating Cylinder Component-model}

The water depth $d[\mathrm{~m}]$, water density $\rho_{w}\left[\mathrm{~kg} / \mathrm{m}^{3}\right]$, density of the mooring line material $\rho_{c}\left[\mathrm{~kg} / \mathrm{m}^{3}\right]$, specific mass of the mooring line in air $m_{a}[\mathrm{~kg} / \mathrm{m}]$, cylinder radius $r[\mathrm{~m}]$, height $h[\mathrm{~m}]$, structural mass $m_{s}[\mathrm{~kg}]$, ballast mass $m_{b}[\mathrm{~kg}]$, vertical centre of gravity position w.r.t. the keel $z_{K G}[\mathrm{~m}]$, added-mass coefficients $C_{m a}^{x}, C_{m a}^{z}[-]$, drag coefficients $C_{D}^{x}$, $C_{D}^{z}$, and damping $C^{x}, C^{z}[\mathrm{~kg} / \mathrm{s}]$ are specified as parameters. $m_{f l g}$ is a parameter to specify if the buoy is free-floating or moored. It is set to 0 in case of a free-floating buoy, and to 1 if moored.

A composite connector Fairlead[2], having two flanges of type Modelica.Mechanics.Translational.Interfaces.Flange_a, is specified at the centre of the bottom surface of the buoy, to transfer the horizontal and vertical mooring loads.

A data connector ebdc is specified to link the wave and current data from the EnvironmentBus to the buoy component-model.

Figure 3 shows the flow-chart for the componentmodel. Here, $K[\mathrm{~N} / \mathrm{m}]$ is the stiffness, $M[\mathrm{~kg}]$ is the dry mass of the buoy, $A_{w p}\left[\mathrm{~m}^{2}\right]$ is the water-plane area of the buoy, $z_{s}[\mathrm{~m}]$ is the static draught, $z_{m}[\mathrm{~m}]$ is the draught considering the mooring line length, $z_{c b}[\mathrm{~m}]$ is the $z$ coordinate of the centre of buoyancy, $z_{f b}[\mathrm{~m}]$ is the calmwater $z$ co-ordinate of the body CG. $z_{1}, z_{2}, z_{3}[\mathrm{~m}]$ are the instantaneous $z$ co-ordinates of the body $\mathrm{CG}$, top surface and bottom surface, respectively. $z[\mathrm{~m}]$ is the instantaneous vertical displacement from $z_{f b}$ and $x[\mathrm{~m}]$ is the displacement in the horizontal direction of the body CG, both of which are to be determined from the equations of motion. $v^{x}$ and $v^{z}[\mathrm{~m} / \mathrm{s}]$ are the body velocities in the $x$ and $z$ directions, while $a^{x}$ and $a^{z}\left[\mathrm{~m} / \mathrm{s}^{2}\right]$ are corresponding accelerations. $S S E_{x}[\mathrm{~m}]$ is the instantaneous sea surface elevation at the $x$ co-ordinate of the body CG, $a_{w}\left[\mathrm{~m} / \mathrm{s}^{2}\right]$ is the vertical component of the wave-induced water-particle acceleration, calculated at the instantaneous $\left(x, z_{c b}\right)$ position, and $M_{F}^{x}[\mathrm{~N}]$ is the instantaneous wave-current Morison loading on the buoy. Fairlead[1]. $f$ and Fairlead[2]. $f$ [N] are the horizontal and vertical components of the mooring load, at the the fairlead. $t, T_{\text {sim }}[\mathrm{s}]$ are the current and total simulation times, respectively.

$S S E_{X}$ is calculated using (5). The function wave_awCalculator returns the value of $a_{w}$ calculated using (8), with consideration of the moved kinematic profile. The function morisonForceCydlBuoy returns the value

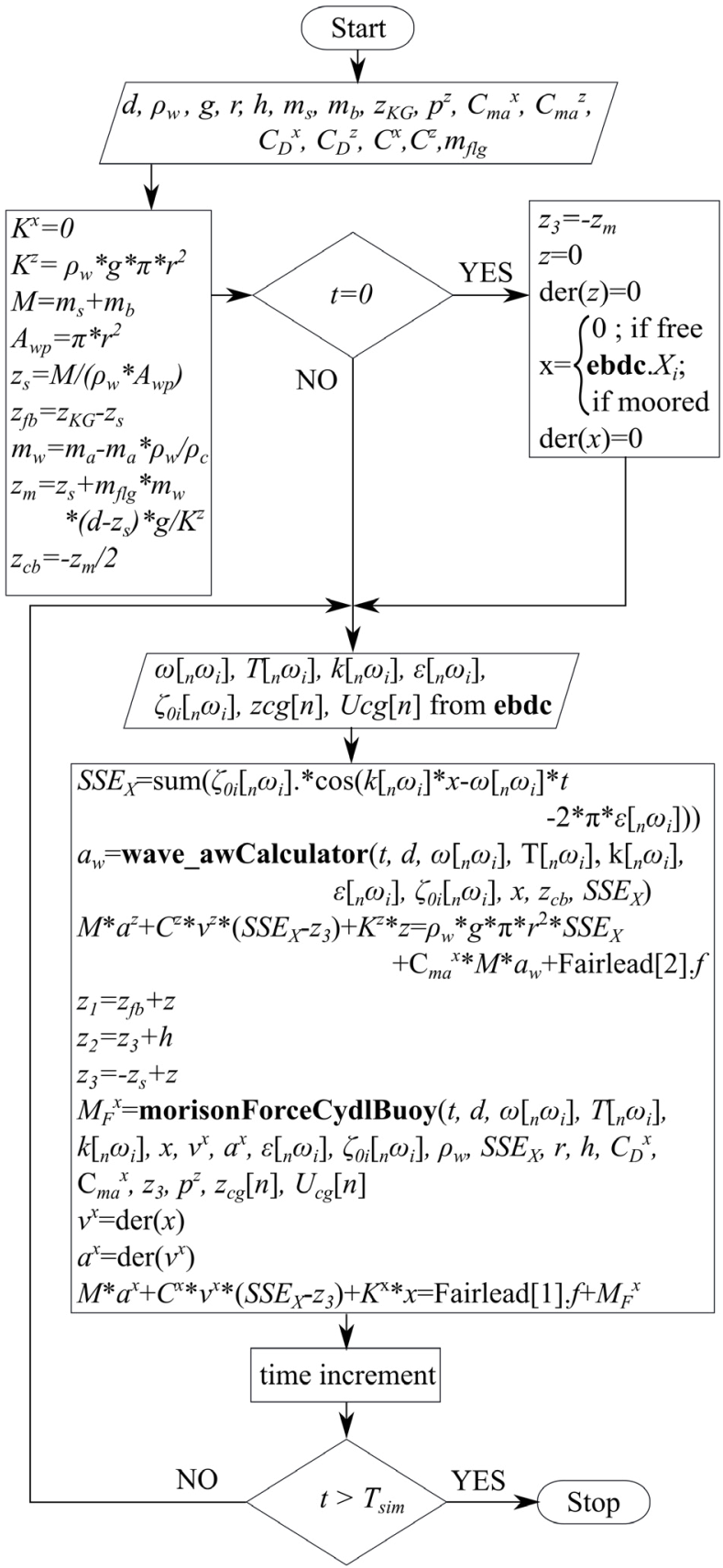

Figure 3. Flow-chart for the floating cylinder componentmodel.

of $M_{F}^{x}$. The EoMs along the $x$ and $z$ directions are then solved with the specified initial conditions to determine the body response.

\subsection{Quasi-static Catenary Mooring Component-Model}

Considering space limitations, the flow chart for the component-model Catenary_Mooring_Mf0, which does not take into consideration the wave and current loads on the mooring line itself, is given in Figure 4. 


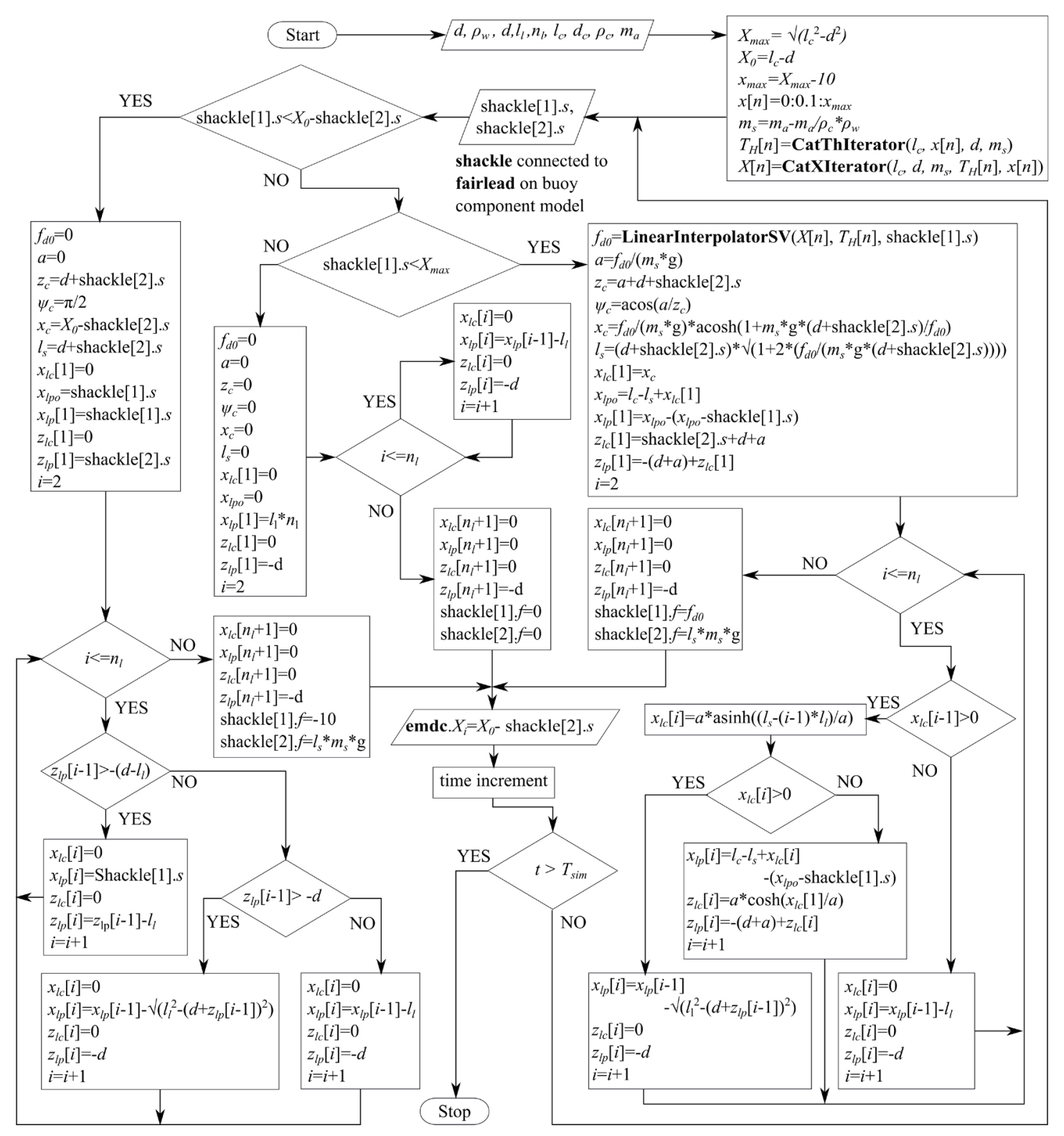

Figure 4. Flow-chart for the quasi-static catenary component-model.

A composite connector

Shackle[2] tor containing the $x$ positions, where the horizontal moorhaving two flanges of type Model- ing load is to be iterated, is defined as $x[n]$. A function ica.Mechanics.Translational.Interfaces.Flange_b, is specified at the top end of the catenary to transfer the horizontal and vertical mooring loads. The parameters defined are the water depth $d[\mathrm{~m}]$, water density $\rho_{w}$ $\left[\mathrm{kg} / \mathrm{m}^{3}\right]$, the number of segments into which the mooring is discretized $n_{l}$, the length of each segment $l_{l}[\mathrm{~m}]$, the density of the mooring material $\rho_{c}\left[\mathrm{~kg} / \mathrm{m}^{3}\right]$, and the dry specific mass of the chain $m_{a}[\mathrm{~kg} / \mathrm{m}]$. Since the Shackle is connected to the Fairlead, the corresponding positional data are also available.

$X_{\max }, X_{0}, x_{\max }[\mathrm{m}]$ are calculated along with the submerged specific mass of the mooring $m_{s}[\mathrm{~kg} / \mathrm{m}]$. The vecCatThIterator returns the vector $T_{H}[n]$, containing the corresponding horizontal tension values, calculated based on (14). Another function CatXIterator, returns the vector $X[n]$ containing the $X$ position corresponding to the $x$ position, as defined in Figure 2.

A data connector emdc is specified to link the wave and current data from the EnvironmentBus to the mooring. In addition, the initial $x$ co-ordinate of the top end of the mooring line $X_{i}[\mathrm{~m}]$, is transmitted to the universal data bus for utilization by the buoy component-model to specify its initial condition.

$f_{d 0}[\mathrm{~N}]$ is the horizontal mooring load, for the given $x$ 
co-ordinate of the top end of the mooring line, $a$ is the catenary parameter, $z_{c}[\mathrm{~m}]$ is the $z$ co-ordinate of the top end of the catenary, in the local co-ordinate system of the catenary, the origin of which lies at a distance of $a$ [m] below the bottom-most point of the catenary, as described in (Tatum, 2004). $\psi_{c}$ [rad] is the slope of the top-most catenary segment, $x_{c}[\mathrm{~m}]$ is the $x$ co-ordinate of the topmost point of the catenary, and $l_{s}[\mathrm{~m}]$ is the suspended length of the catenary.

$x_{l c}$ and $z_{l c}$ are vectors holding the $x$ and $z$ co-ordinates of the end points of the segments, in the local co-ordinate system of the catenary. $x_{l p}$ and $z_{l p}$ are the vectors holding the $x$ and $z$ co-ordinates of the end points of the segments, in the global coordinate system. $x_{l p o}$ is the plot correction parameter to account for the minor difference between the actual catenary shape with its top end $z$ coordinate corresponding to the instantaneous position of the buoy keel, and the catenary shape which is back-calculated based on the horizontal tension value from the look-up table, which is in turn based on the $z$ co-ordinate of the top end of the catenary lying at the sea-surface.

If the $x$ co-ordinate of the shackle is less than $X_{\min }[\mathrm{m}]$, then a small force in the positive $x$ direction is applied to restore the buoy to a region where the mooring model is valid. The vertical mooring load is then the weight of the vertically suspended length of the mooring. For the rare cases when shackle[1].s $<X_{\text {min }}$, the plot of the mooring shows a vertically suspended-length instead of the actual shape.

When the $x$ co-ordinate of the shackle is between $X_{\text {min }}$ and $X_{\max }$, the horizontal mooring load is the corresponding value interpolated from the lookup-table using a function linearInterpolatorSV, and the vertical load is based on the suspended length back-calculated from the horizontal load. The plot of the mooring line shows the catenary shape, back-calculated from the horizontal load, and corrected using $x_{l p o}$.

If the loads on the buoy exceed the capacity of the mooring line, then the $x$ co-ordinate of the shackle exceeds $X_{\text {max }}$, the catenary is assumed to be detached from the buoy, and would lie extended on the sea-floor.

\subsubsection{Current and Wave Loads on the Mooring Line}

Simulation of current and wave Morision loads on the mooring line is based on the theory given in Section 2.2. The methodology is similar to the one represented by Figure 4, with additional loops for determining fluid and structure velocities and accelerations, and is easily discernible from the code. Catenary_Mooring_MfC considers the Morison loads due to current and mooring velocities, while Catenary_Mooring_MfCW considers the loads due to current, wave, and mooring line velocities and accelerations.

\section{Results}

The simulation files for all results discussed below are available at github.com/Savin-Viswanathan/
Modelica2020-b. Comparison results using Orcaflex are also presented here.

Figure 5a shows the heave response of a cylindrical buoy of $r=0.6[\mathrm{~m}], h=2[\mathrm{~m}], m_{s}=350[\mathrm{~kg}], m_{b}=500$ $[\mathrm{kg}]$, in a water depth of $d=50[\mathrm{~m}]$, when subjected to a regular wave with $H_{r}=1[\mathrm{~m}]$ and $T_{r}=7$ [s], with $T_{d e l}=0$ [s], and $T_{r m p}=20[\mathrm{~s}]$. We have assumed $C_{m a}^{x}=C_{m a}^{z}=1$, $C^{x}=0[\mathrm{~kg} / \mathrm{s}]$, and $C^{z}=3100[\mathrm{~kg} / \mathrm{s}]$.

Figure (4) in (Viswanathan and Holden, 2019) had shown the same results for heave, and we had noticed a slight discrepancy between the Modelica and Orcaflex results. The cause was identified to be an error in the treatment of the added mass term in (4) of (Viswanathan and Holden, 2019), and has been corrected based on the theory described here in Section 2.1.1.1. Figure 5b shows the surge response.

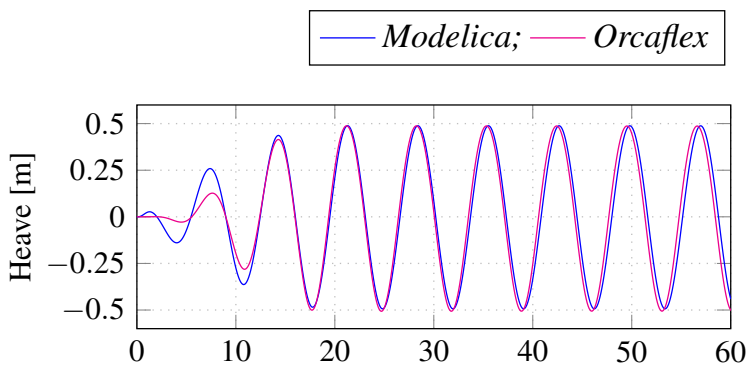

(a) Heave response.

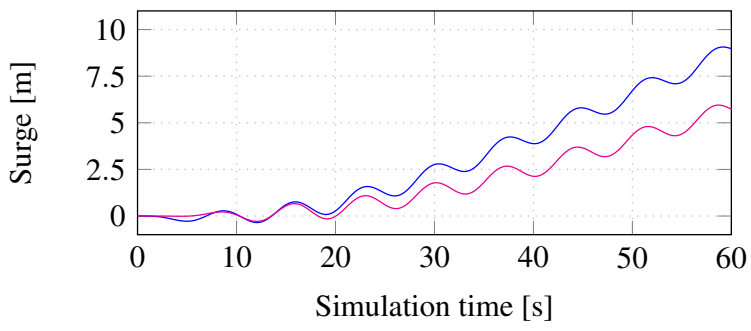

(b) Surge response.

Figure 5. Unmoored cylindrical buoy in waves.

Figure 6a shows the surge response of the above buoy, in the presence of a uniform current of $1[\mathrm{~m} / \mathrm{s}]$ in the $x$ direction, while Figure $6 \mathrm{~b}$ shows the same in presence of both the above wave and current.

Figure 7a shows the horizontal Morison loads on a fixed buoy with same properties as the earlier one, but with a draught of $1[\mathrm{~m}]$, when subjected to a uniform current of $1[\mathrm{~m} / \mathrm{s}]$. Figure $7 \mathrm{~b}$ shows the surge Morison loads when only a regular wave, with the same parameters as above, acts on the fixed buoy, and Figure 7c shows the Morison loads when both the current and the wave acts on the buoy.

From Figure 7, we observe that the Morison loads are a close match, and hence, the difference between Modelica and Orcaflex values in Figures 5b, 6a, and 6b, can be attributed to the difference in the way in which the loads are ramped up during the start of simulation, as evid- 
Modelica; - Orcaflex

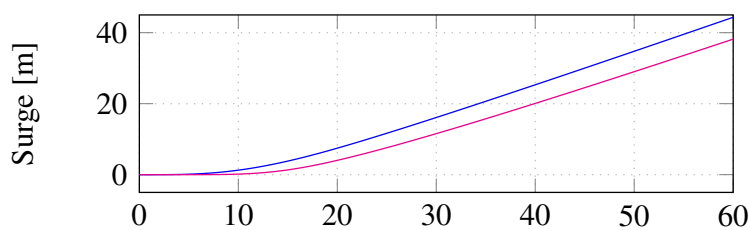

(a) Current only.

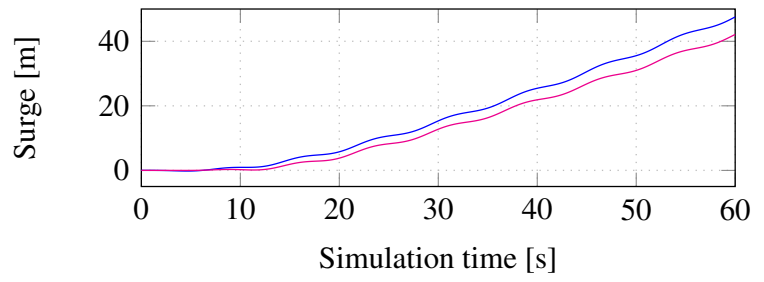

(b) Wave and current.

Figure 6. Surge response of an unmoored cylindrical buoy.
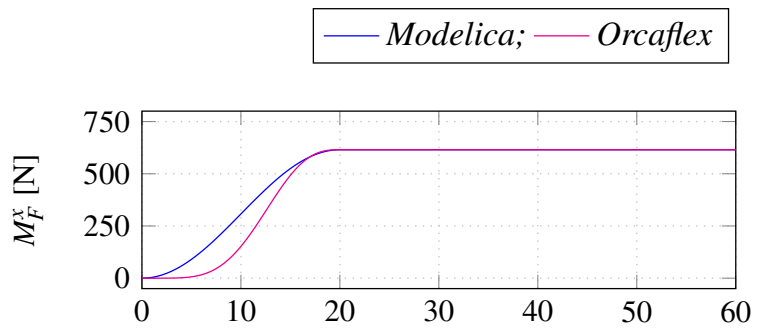

(a) Current only.

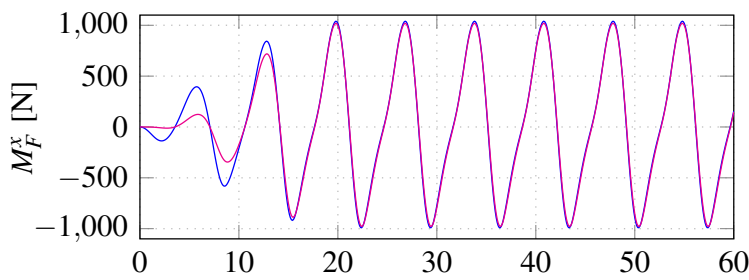

(b) Wave only.

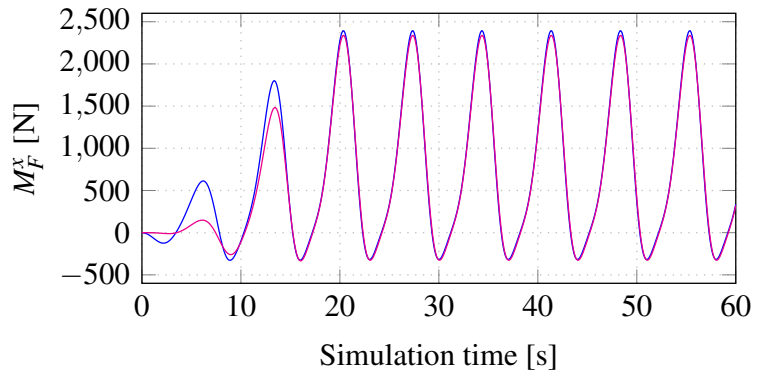

(c) Waves and current.

Figure 7. Morison loads on a fixed cylinder.

ent from Figure 7a. Orcaflex uses vertical-stretching of the water-particle kinematics, while the present Modelica model employs moved kinematic-profiles, and this could be the cause of the minute difference in peak values of the Morison loads in Figures $7 \mathrm{~b}$ and $7 \mathrm{c}$.

Orcaflex uses a lumped-mass and spring-damper model for the mooring lines, while mooring forces in the present work are based on the quasi-static catenary theory. Figure 8 shows the horizontal tension values given by $\mathrm{Or}$ caflex and Modelica models for different $X$ positions, for moorings of different specific masses. The horizontal tensions are a close fit, with Modelica giving slightly higher values than Orcaflex. e.g., for a chain with specific mass of $16[\mathrm{~kg} / \mathrm{m}]$, at $X=70[\mathrm{~m}], T_{H}$ in Modelica is 2,336 [N] and 2,299 [N] in Orcaflex. The mooring horizontal tensions from Orcaflex were determined by placing the top end of the line at different $X$ positions along the free surface manually and a small error in the values had occured in Figure 10 of (Viswanathan and Holden, 2019).

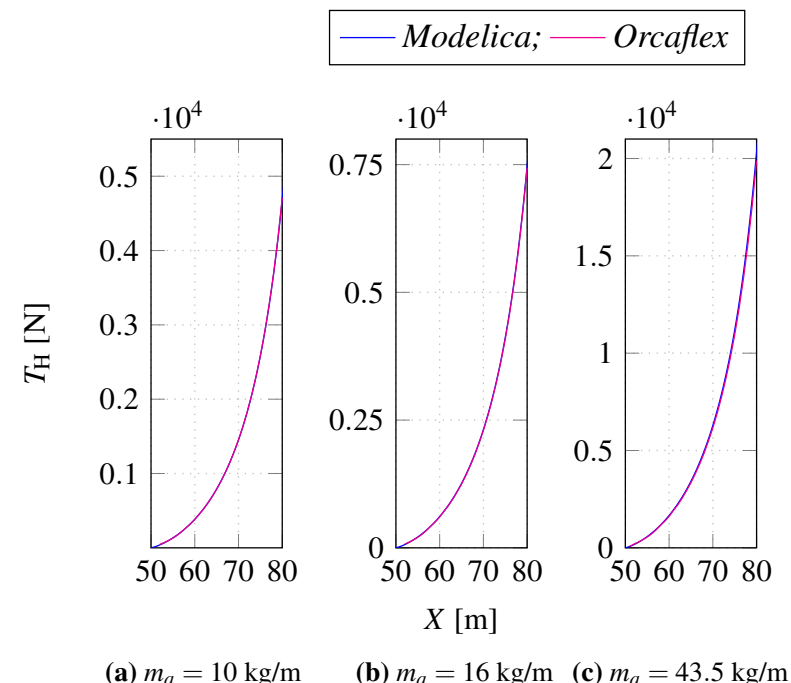

Figure 8. Horizontal tensions for mooring chains with different specific masses $\left(m_{a}\right)$.

Figure 9 shows the shape of a mooring line, with specific mass $10[\mathrm{~kg} / \mathrm{m}]$, in Modelica and Orcaflex when the top end is placed at $X=60[\mathrm{~m}]$, and at $80[\mathrm{~m}]$ with $z=0[\mathrm{~m}]$. When a uniform current of $1[\mathrm{~m} / \mathrm{s}]$ is applied across the full depth of the water-column, the Orcaflex line, based on the lumped mass model, deflects under the influence of the current. The deflection is not expected to be large enough to cause considerable difference in the fluid loading experienced by the mooring line. However, it is evident that the static catenary model would not capture the forces that result as a consequence of the dynamics of the mooring line itself.

Figure 10 shows the surge and heave response of the above free-floating buoy, when moored with a mooring line of specific mass $10[\mathrm{~kg} / \mathrm{m}]$, under different conditions of wave and current. It was noticed that the model failed to simulate when the acceleration forces due to the fluid and the motion of the chain were considered, using the CatenaryMooring_MfCW component model. Hence the 


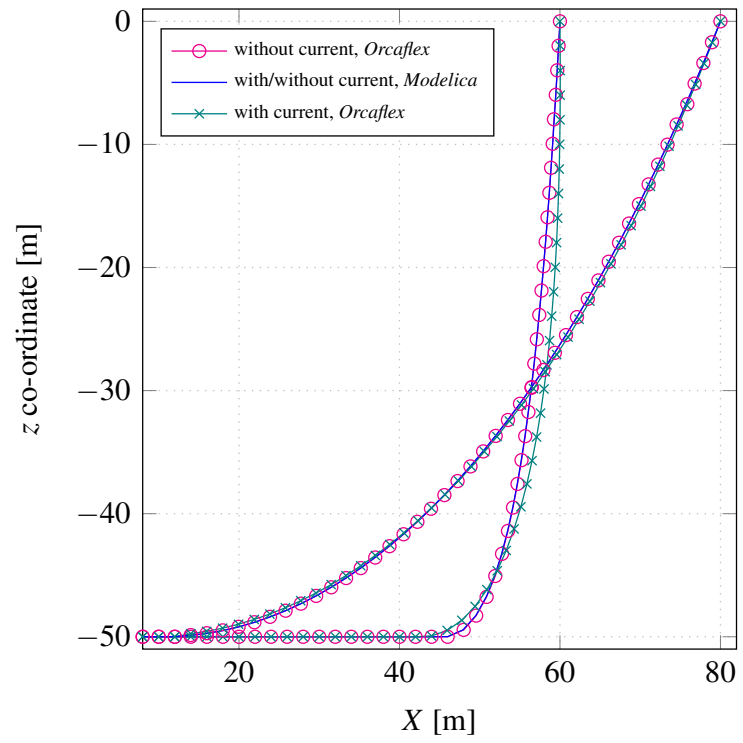

Figure 9. Shape of the mooring line.

results shown are with the CatenaryMooring_MfC component model. The combined wave-current velocity and accleration loads, and inertial loads due to the structural response of the mooring chain, are inherent in the Orcaflex model, while they are not accounted for by the present Modelica model. The phase difference in the response between the models may be because of this difference.

When the current velocity is reduced to $0.5[\mathrm{~m} / \mathrm{s}]$, CatenaryMooring_MfCW could be used for simulation, and the results are shown in Figure 11. However, the model is sluggish with many warnings for nonconvergence. This could be due to the discontinuities in the accelerations of the segment mid-points, calculated based on the instantaneous static catenary shape. An example of the vertical component of the acceleration of the mid-point of the second segment from the top-end of the mooring, is shown in Figure 11c.

\section{Conclusion}

Implementation of the theory to develop Modelica component-models for a non-diffracting cylindrical object, and for a quasi-static mooring catenary, is described in detail. Simulations to determine the hydrodynamic response of a free-floating cylinder are carried out, and the results compared with a smilar model in Orcaflex. It is observed that the heave responses in both cases are in satisfactory agreement. Minor differences in the surge responses are reconciled based on the comparison of Morison forces on a fixed cylinder, under various loading scenarios, and it is concluded that these differences are a result of the differences in the ramp-up functions used in this Modelica model and in Orcaflex, and hence, do not constitute errors in the simulation results.

The static mooring loads, based on the catenary the-

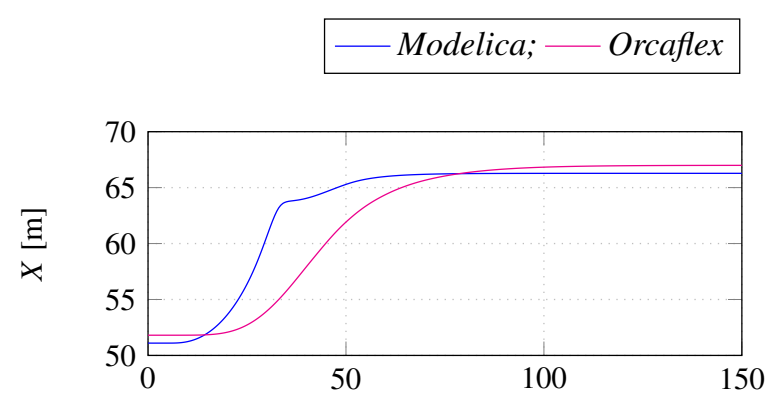

(a) Surge response to current.

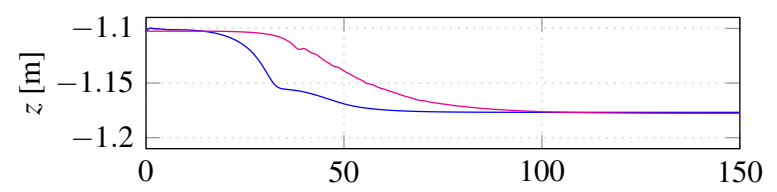

(b) Heave response (keel) to current.

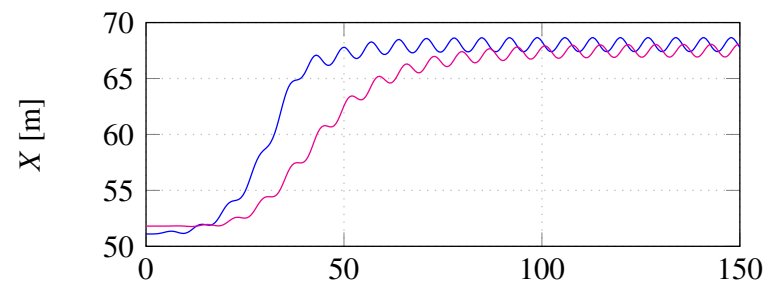

(c) Surge response to waves and current.

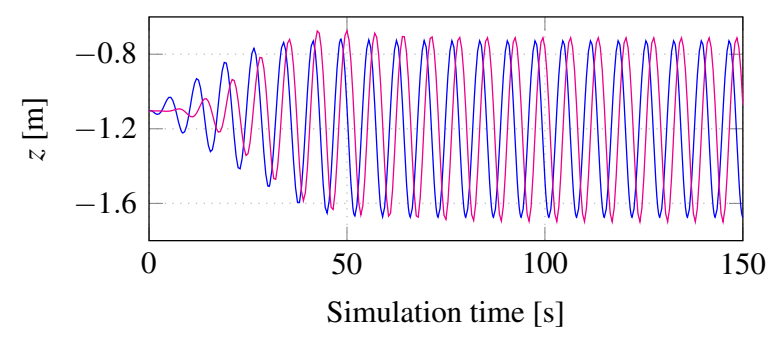

(d) Heave response (keel) to waves and current.

Figure 10. Hydrodynamic response of a moored cylindrical buoy.

ory in the present Modelica model, and those based on the lumped-mass spring-damper system of the Orcaflex model, are demostrated to have satisfactory agreement. The comparison between mooring configurations under different loading scenarios points out the probability that differences in the fluid loading of the mooring line, as a result of the deviation of the mooring line from the catenary shape, might not be significant, compared with the contributions from the dynamics of the mooring line itself, which the present Modelica model does not capture.

The simulations of a moored floating cylinder further demostrate the satisfactory agreement between this Modelica model and a similar Orcaflex model. The simulations bring out the deficiencies caused by the assumptions of the quasi-static catenary, which is not in agreement with the actual physics of the system. 


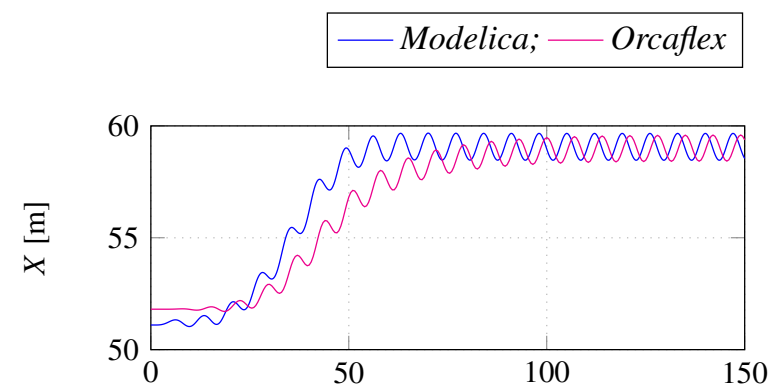

(a) Surge response.

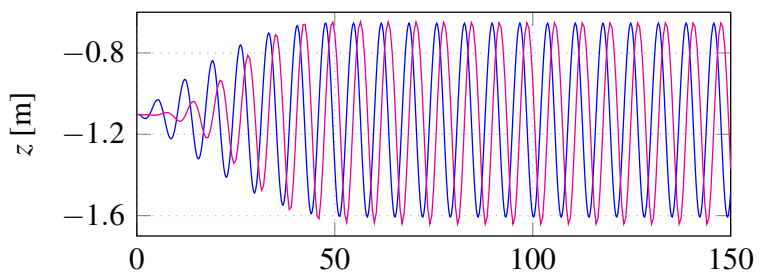

(b) Heave response (keel).

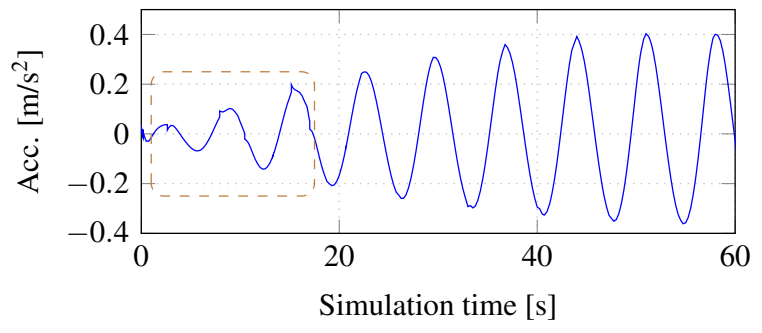

(c) Vertical acceleration of the second chain-link from the fairlead.

Figure 11. Moored cylindrical buoy in waves and reduced current.

To overcome the deficiency of not being able to account for the mooring line dynamics, and to mitigate issues as seen in Figure 11c, a Modelica mooring component-model based on the lumped-mass spring-damper approach is being developed, along with a frequency-domain hyrdodynamic analysis component-model, which would enable the generation of hydrodynamic parameters for diffracting objects. The initial results appear promising and will be the topic of discussion in a future work.

\section{Acknowledgements}

The research in this paper has received funding from the Research Council of Norway, SFI Offshore Mechatronics, project number 90034210 .

\section{References}

Subratha Kumar Chakrabarti. Hydrodynamics of Offshore Structures. Computational Mechanics Publications, and SpringerVerlag, Dorchester, Great Britain, 1987. ISBN 0-905451-66$\mathrm{X}$.

O.M. Faltinsen. Sea Loads on Ships and Offshore Structures. Cambridge University Press, 1999. ISBN 0-521-45870-6.
O.M. Faltinsen and F.C. Michelsen. Motion of large structures in waves at zero froude numbers. Read at the International Symposium on the Dynamics of Marine Vehicles and Structures in Waves, London, 1974.

MIT. Lecture Notes on Mooring Dynamics-II. Massachusetts Institute of Technology, 2011. URL Ocw. mit.edu/courses/mechanical-engineering/ 2-019-design-of-ocean-systems-spring-2011/ lecture-notes/MIT2_019S11_MD2.pdf.

J. N. Newman and C. H. Lee. Boundary-element methods in offshore structure analysis. Journal of Offshore Mechanics and Arctic Engineering, 124:81-89, May 2002. doi:10.1115/1.1464561.

J.N Newman. Marine Hydrodynamics. The MIT Press, Cambridge, Massachusetts, 1989. ISBN 0-262-14026-8.

Orcina. Orcaflex Manual- Version 9.1a. Orcina, 2010. URL citeseerx.ist.psu.edu/viewdoc/download? doi=10.1.1.121.721\&rep=rep $1 \&$ type=pdf.

Yan-Lin Shao and O.M. Flatinsen. A harmonic polynomial cell (hpc) method for 3d laplace equation with application in marine hydrodynamics. Journal of Computational Physics, (274):312-314, 2014.

SINTEF. Handbook on Design and Operation of Flexible Pipes. 2014. URL sintef.no/en/latest-news/ updated-handbook-on-design-and-operationof-flexible-pipes/.

Jeremy B. Tatum. Lecture Notes on the Catenary. University of Victoria, 2004. URL astrowww . phys . uvic. ca/ tatum/classmechs/class18.pdf.

A.H. Techet. Lecture Notes on the Design Principles for Ocean Vehicles. Massachusetts Institute of Technology, 2005. URL ocw.mit.edu/courses/ mechanical-engineering/2-22-designprinciples-for-ocean-vehicles-13-42spring-2005/readings/r10_froudekrylov. pdf.

Savin Viswanathan and Christian Holden. Towards the development of an ocean engineering library for openmodelica. In Proceedings of the ASME 2019 38th International Conference on Ocean, Offshore and Arctic Engineering., volume 7B: Ocean Engineering, OMAE2019-95054, June, 2019. URL doi .org/10.1115/OMAE2019-95054.

Savin Viswanathan and Christian Holden. Modelica componentmodels for oceanic surface-waves and depth varying current. Proceedings of the American Modelica Conference, March, 2020. The referring paper and the referred paper are part of the proceedings of the same conference.

Ronald W. Yeung. Added mass and damping of a vertical cylinder in finite-depth waters. Applied Ocean Research, 3(3): 119-133, 1981. 